Buenos Aires, Argentina. E-mail: rhcaraballo@arnet.com.ar).

COMMENT. Childhood occipital epilepsy (COE) of Gastaut is manifested by brief seizures, mainly visual hallucinations, illusions or amaurosis, followed by hemiclonic seizures while awake, postictal migraine headaches, mean age at onset of 8.9 years, and interictal occipital spike-wave EEG paroxysms that attenuate when eyes are opened. Prevalence is estimated at $0.2-0.9 \%$ of epilepsies, and $2-7 \%$ of benign childhood focal seizures. Gastaut reported the syndrome in 1982, and it was accepted as an entity by the ILAE in 1989. Despite some recent loss of recognition, the present authors consider the syndrome as a rare but well-defined entity within the group of idiopathic simple partial (focal) epilepsies of childhood. Differential diagnoses include symptomatic occipital epilepsy, migraine with aura, and basilar migraine. Among the idiopathic COEs, the Gastaut type is of late onset and associated with visual symptoms, whereas Panayiotopoulos syndrome is of early onset and characterized by autonomic symptoms (ictal vomiting). The EEG findings alone are not diagnostic of COE. Not all children with occipital spikes develop seizures. Those with $\mathrm{COE}$ should be differentiated from patients with occipital spikes occurring both with eyes open and closed, and unassociated with clinical seizures. Occipital spikes also occur in children with myoclonic, absence, and photosensitive epilepsies. (Browne TR, Holmes GL Handbook of Epilepsy. Philadelphia. Lippincott, 2004;86-87).

\title{
RISK FACTORS FOR FEBRILE SEIZURE RECURRENCE
}

Factors that predict recurrence of febrile seizures (FS) were determined in a prospective study of 260 children age 3 months to 6 years followed for a median of 4.3 years after the first FS at Ippokratio Hospital, Aristotle University of Thessaloniki, Greece. The median age of patients at onset of study was 16.5 months (range 3 months to 5.8 years). Final reevaluation was at a median of $6.0 \mathrm{t} / \mathrm{-} 1.5$ years. The sex ratio was 139 boys to 121 girls $(1.15: 1)$, and recurrence was not higher among boys than girls. Overall recurrence rate was $40.4 \%$. Cumulative recurrence was $24.2 \%$ at 6 months, $34.2 \%$ at 12 months, $38.1 \%$ at 18 months, and $40.4 \%$ at 6 years. EEG abnormalities in 12 children at baseline were not significantly associated with FS recurrence. Low age at onset and positive family history of FS, especially maternal, were strong predictors of recurrence. Other risk factors included abnormal perinatal history with low Apgar score and NICU care for $>3$ days, low temperature (38.9C or below) and short $(<12 \mathrm{hrs})$ duration of fever before initial FS, a history of frequent febrile illness $(\mathrm{p}<0.0001)$, focal FS, and recurrence within the same febrile illness. Duration $>15$ min of first FS was not a factor. Two or more recurrences occurred in $48 \%$; 28 had only 2 and $22(44 \%)$ had 3 or more recurrences. Factors predisposing a child with one recurrence to a second or more are young age at onset and family history of FS $(\mathrm{p}<0.001)$. Multiple recurrences were correlated with low temperature elevation $(</=38.9 \mathrm{C})$ before initial FS. By multivariate analysis significant risk factors were early age at onset, complex first FS, and family history of FS $(\mathrm{p}<0.05)$. (Pavlidou E, Tzitiridou M, Kontopoulos E, Panteliadis CP. Which factors determine febrile seizure recurrence? A prospective study. Brain Dev Jan 2008;30:7-13). (Respond: Dr Christos P Panteliadis, Department o Paediatric Neurology, Ippokratio Hospital, Aristotle University of Thessaloniki, Greece. E-mail: cpantel@hol.gr). 
COMMENT. In confirmation of previous studies, the main predictors of susceptibility to febrile seizure recurrence are young age $(<18 \mathrm{mo})$ at initial FS, family history of FS in first degree relative, lower temperature $(</=38.9 \mathrm{C})$ and shorter fever duration $(<12 \mathrm{hrs})$ before initial seizure. The lower temperature indicates a low threshold to FS. The present authors also found a maternal preponderance in the families of children with FS recurrence. Daycare attendance and frequent viral infection are additional risks.

Cytokines in acute encephalopathy following prolonged febrile seizures. In a study of 13 children with acute encephalopathy following prolonged febrile seizures compared to 23 without encephalopathy, in Yamaguchi University and other centers in Japan, serum IL-6, IL-10, TNFR1 and CSF IL-6 levels were significantly higher in subjects with encephalopathy compared to controls without encephalopathy. The authors speculate that IL6 is induced in the CNS to protect damaged brain following prolonged febrile seizure.

\section{PERTUSSIS VACCINATION, EPILEPSY AND SCN1A MUTATION}

Literature regarding pertussis vaccination and risk of encephalopathy and/or epilepsy is reviewed by researchers from UCL Institute of Neurology, London, UK, and North Illinois University, DeKalb, IL, USA. Current risk estimates of vaccine-related febrile seizure are 1 per 18,496 vaccinations; afebrile seizure 1 per 76,133 ; and encephalopathy $0-3$ per million. The rate of febrile seizures within 2 days of the present acellular vaccine is much lower than that of 1 per 2835 with previous whole-cell vaccine (Cody CL et al. Pediatrics 1981;68:650$660)$.

As part of a recent study of unexplained encephalopathies in Australia, New Zealand, Canada and Scotland, Berkovic SF et al (Lancet Neurol 2006;5:488-492) identified 14 cases within 72 hours of pertussis vaccination, treated by child neurologists. Of these presumed vaccine-related cases, 11 had an inherited genetic defect of the SCN1A gene that corresponded to the phenotype for severe myoclonic epilepsy of infancy (SMEI, Dravet syndrome). The encephalopathy temporally associated with pertussis vaccination may, in some cases, be due to an SCNIA mutation and Dravet syndrome. This finding requires replication by further studies. (Shorvon S, Berg A. Pertussis vaccination and epilepsy - an erratic history, new research and the mismatch between science and social policy. Epilepsia Feb 2008;49:219-225). (Respond: Dr Simon Shorvon, UCL Institute of Neurology, Box 5, Nationai Hospital for Neurology and Neurosurgery, Queen Square, London, UK).

COMMENT. Fortunately, the risk of neurologic complications of pertussis vaccine has fallen remarkably since the introduction of the acellular vaccine. With whole-cell vaccine, reports of neurologic complications, especially febrile seizures or infantile spasms, were not uncommon in US pediatric neurology practice. In the same time period, encephalopathy attributed to whooping cough was a very rare neurologic diagnosis. In vaccine related encephalopathies, a test for SMEI should be considered in the differential diagnosis.

In Australia and New Zealand, the experience is different from the US. Among 49 unimmunized infants (age 6 weeks) admitted with pertussis to the PICU at Royal Children's Hospital, Melbourne, between 1985-2004, 63\% had apnea, 18\% pneumonia, and 10\% had seizures. Deaths in 7 were due to pneumonia and circulatory failure. (Namachivayam P et al. 